\title{
Toxicological Examinations Following Oral Administration of Bacillus thuringiensis var kurstaki Biological Insecticide in Wistar Rats
}

\author{
Reza Abbasi Larki ${ }^{1}$, Ehsan Zayerzadeh ${ }^{2 *}$, Naser Harzandi ${ }^{1}$, Ali Anissian ${ }^{3}$ \\ 'Department of Microbiology, Karaj Branch, Islamic Azad University, Karaj, Iran \\ ${ }^{2}$ Department of Biology, Faculty of Food Industry and Agriculture, Standard Research Institute, Karaj, Iran \\ ${ }^{3}$ Department of Veterinary Medicine, Islamic Azad University, Abhar, Abhar Branch, Iran
}

Corresponding Author: Ehsan Zayerzadeh, PhD, Assistant Professor, Department of Biology, Faculty of Food Industry and Agriculture, Standard Research Institute, Karaj, Iran. Tel: +98-9129237820, Fax: +98-26-22867922, Email: zayerzadeh@standard.ac.ir

Received August 5, 2018; Accepted December 26, 2018; Online Published March 15, 2019

\begin{abstract}
Introduction: Bacillus thuringiensis var kurstaki is one of the best known biological insecticide which is used extensively in the world. This biopesticide has been broadly used against important insect pests and vectors of animals and humans. However, the controversial data was published to date considering toxicological studies on non-target species are urgent to determine probable adverse effects and risk assessment of this biopesticide. In this research, histopathological changes, hematological and some biochemical factors were evaluated following the single dose oral administration of $B$. thuringiensis in rats.

Materials and Methods: Twelve Wistar rats were randomly divided into 2 groups including experimental and control groups. Animals were treated orally by gavage to single dose of sub-lethal dose $(5000 \mathrm{mg} / \mathrm{kg}$ ) of B. thuringiensis suspension. Finally, hematological factors (WBC, RBC, Hb, HCT, MCV, MCH, MCHC and PLT), some biochemical parameters (ALT, AST, ALP, BUN and Cr) and histopathological observations were evaluated.

Results: The results demonstrated that oral administration of high dose of $B$. thuringiensis biopesticide induced some pathological complications including congestion and inflammation in vital organs of rats such as liver, heart, lung and kidney. It also caused hematological abnormalities and biochemical alterations in the experimental animals group.

Conclusions: According to the findings, it can be concluded that high dose of $B$. thuringiensis biopesticide can induce toxicity in rats. Therefore, further investigations including subacute and chronic are recommended.

Keywords: Bacillus Thuringiensis, Biological Pesticide, Cry Toxin, Pathology, Hematology, Rats

Citation: Abbasi Larki R, Zayerzadeh E, Harzandi N, Anissian A. Toxicological examinations following oral administration of Bacillus thuringiensis var kurstaki biological insecticide in Wistar rats. J Appl Biotechnol Rep. 2019;6(1):34-39. doi:10.29252/JABR.06.01.06.
\end{abstract}

\section{Introduction}

Potential health adverse effects following exposure to pesticides have raised the consideration of alternative safer methods for pest control. Fifty years ago, biological insecticides such as microbial pesticidees have received specific attention as environmentally safer alternatives. ${ }^{1}$ Bacillus thuringiensis is one of the well-known and the most widely used biological pesticides. $^{2}$ This biopesticides can produce crystalline, proteinaceous and delta-endotoxin, which are highly toxic for different types of agricultural pests.,4 The B. thuringiensis toxins have massive potential for the control of agricultural diseases. ${ }^{5}$ They target a limited spectrum of pest insects that contain specific physiological properties (i.e., gut $\mathrm{pH}$ and toxin receptor sites in the midgut) and eventually have low risk to non-target species than broad-spectrum insecticides. ${ }^{6-8}$ Cry toxins are mostly found in $B$. thuringiensis strains that mediate their toxic effects on a target organism. ${ }^{9}$ Following ingestion by larval insects, the crystals were solubilized in the midgut and subsequently activated by proteases. The activated toxins bind to midgut epithelial cells, form pores and cause osmotic cell lysis eventually leading to insect death. Different B. thuringiensis toxins and B. thuringiensis toxin genes have been commercially produced for the control of Lepidoptera, Diptera, and other insect orders. ${ }^{10-12}$ However, oral exposure of this microbial pesticide is one of the major routes of human and animal exposure due to extensive application of this product in agricultural crops in Iran. Of course, human exposure during the manufacturing of this product is inevitable. On the other hand, there are concerns regarding the probable health effects of Cry toxins on vertebrates, particularly $B$. thuringiensis toxins with inducing toxic responses. Thus, the controversial results that were published considering earlier toxicological studies of $B$. thuringiensis, spores, toxins and B. thuringiensis crops on mammals are urgent to determine adverse effects of this biopesticide ensuring human and environmental biosafety. ${ }^{9}$

Copyright $\odot 2019$ The Author(s). This is an open-access article distributed under the terms of the Creative Commons Attribution License (http:// creativecommons.org/licenses/by/4.0), which permits unrestricted use, distribution, and reproduction in any medium, provided the original work is properly cited. 
To our knowledge, there is no animal study which evaluates toxicity of $B$. thuringiensis microbial pesticide formulated in Iran. Therefore, in the present research, the oral toxicity of $B$. thuringiensis microbial insecticide in rat model was studied according to the histopathological, hematological and biochemical analysis. The results of the present study will be a fundamental step for the risk assessment of B. thuringiensis microbial insecticide in Iran.

\section{Materials and Methods}

Test Materials

Spore-crystals in the lyophilized form of Bacillus thuringiensis var kurstaki strain PTCC1531 (Cry1A and Cry1C insecticidal toxins) were obtained from the market in Iran (wettable powder, stability: 2 years).

\section{Animals}

In the present study, 12 adult male Wistar rats weighing 220 $\pm 5 \mathrm{~g}$ were obtained from the Pasture Institute of Iran. All rats were housed in separate cages and were allowed to be adapted with a lab environment before the experiment. After seven days adaptation with the laboratory environment, rats were randomly allocated into 2 groups. They were kept under sterile and standard conditions (temperature of $22 \pm 2{ }^{\circ} \mathrm{C}$, humidity $55 \pm 5$, and a 12:12 light/dark cycle) with adequate standard laboratory food and tap water. All animals were kept in according to the recommendation of the animal care committee of the Tehran University based on the 'Guide for Care and Use of Laboratory Animals' (NIH US publication 86-23, revised 1985).

\section{Treatment}

Bacillus thuringiensis biopesticide were suspended in a sterile saline solution. Twelve rats were randomly divided into 2 groups (6 for each group). One group was chosen as the saline control (first group) and the second group was used as the dosing group. Single sublethal dose (5000 mg/kg BW) of $B$. thuringiensis biopesticide suspension was administered orally to the experimented group of animals. In the pilot experiments, exposures greater than $5000 \mathrm{mg} / \mathrm{kg}$ had caused signs of toxicity, so this concentration was considered the maximum tolerated. Finally, after 2 weeks, the animals were sacrificed by intraperitoneal injection of ketamine (30-50 mg/ $\mathrm{kg}$ ) and xylazine (3-5 mg/kg) following the single dose oral administration of this biological insecticide.

\section{Hematological assessment}

Hematological parameters were performed using a hemocytometer for veterinary use (ADVIA 120, Hematology system, Siemens, Germany) and was calibrated for rat, in microtubes containing EDTA as anticoagulant. The results represent the mean \pm standard deviation (SD) per percentage of cell suspension.

\section{Evaluation of Biomarkers}

Blood samples were collected from ventricle into test tubes containing EDTA, kept for 30 minutes at lab temperature and centrifuged at $3000 \mathrm{rpm}$ for 20 minutes. After that, serum samples were separated and the levels of blood urea nitrogen (BUN), creatinine, alanine aminotransferase (ALT), aspartate aminotransferase (AST) and alkaline phosphatase (ALP) were measured using the auto analyzer (BT3000, Italy) and (Biosystems kits, Spain) according to the manufacturer's instructions.

\section{Histological Analysis}

The heart, lungs, kidney and liver were isolated and immersed in $10 \%$ buffered formalin for 48 hours at room temperature and then sectioned transversely in 3-4 $\mu \mathrm{m}$ slices. Samples were dehydrated in a graded series of alcohol and xylene and embedded in paraffin. For histological processing, the sectioned tissues were stained with Hematoxylin-Eosin and examined for morphological and histological parameters by light microscope (Labomed Lx 400, USA).

\section{Statistical Analysis}

All data were expressed as mean \pm SD. The mean of all the parameter between the two groups were compared using the Student's $t$ test. Data were analyzed using the SPSS software (version 19) and $P<0.05$ was considered statistically significant.

\section{Results}

Hematological Assessment

The means of hematological findings were demonstrated in Table 1. The means of parameters including white blood cell (WBC), red blood cell (RBC), hemoglobin ( $\mathrm{Hb})$, hematocrit (HCT), mean corpuscular hemoglobin (MCV) and platelet (PLT) in the treatment group were significantly raised. On the other hand, the means of other hematological parameters including $\mathrm{MCH}$ and $\mathrm{MCHC}$ in this group did not show any significant alterations compared to the control group.

\section{Biomarkers Assessment}

Serum ALT, AST and ALP activity were significantly increased in the experimented group compared to the control group. However, serum BUN and creatinine level were not significantly changed in this group (Table 2).

Table 1. Hematology Parameters Changes Following Oral Administration of Bacillus thuringiensis var kurstaki Biopesticide (5000 mg/kg) in Rats

\begin{tabular}{lcc}
\hline \multirow{2}{*}{ Parameters } & \multicolumn{2}{c}{ Groups } \\
\cline { 2 - 3 } & First group & Second group \\
\hline WBC $\left(10^{3} / \mu \mathrm{L}\right)$ & $8.63 \pm 1.32$ & $15.08 \pm 3.97^{*}$ \\
\hline $\mathrm{RBC}\left(10^{6} / \mu \mathrm{L}\right)$ & $6.70 \pm 0.97$ & $7.86 \pm 0.13^{*}$ \\
\hline $\mathrm{Hb}(\mathrm{g} / \mathrm{dL})$ & $12.05 \pm 1.51$ & $15.3 \pm .643^{*}$ \\
\hline $\mathrm{HCT}(\%)$ & $33.6 \pm 5.80$ & $44.0 \pm 1.32^{*}$ \\
$\mathrm{MCV}(\mathrm{FL})$ & $50.56 \pm 1.50$ & $55.91 \pm 1.62^{*}$ \\
\hline $\mathrm{MCH}(\mathrm{pg})$ & $18.01 \pm 0.972$ & $19.55 \pm 0.683$ \\
$\mathrm{MCHC}(\mathrm{g} / \mathrm{dL})$ & $34.18 \pm 1.16$ & $34.43 \pm 1.18$ \\
\hline $\mathrm{PLT}\left(10^{5} / \mu \mathrm{L}\right)$ & $578.83 \pm 87.01$ & $781.83 \pm 89.82^{*}$ \\
\hline
\end{tabular}

Mean values (standard deviation) are shown for the 6 animals in each group. *Significant difference $(P<0.05)$.

First group: control group. Second group: $B$. thuringiensis treatment group. 
Table 2. AST, ALT, ALP, BUN and Creatinine Activity Following Oral Administration of Bacillus thuringiensis var kurstaki Biopesticide (5000 mg/kg) in Rats

\begin{tabular}{lccccc}
\hline \multirow{2}{*}{ Groups } & \multicolumn{3}{c}{ Biomarkers } \\
\cline { 2 - 5 } & ALT (U/L) & AST (U/L) & ALP (U/L) & BUN (mg/dL) \\
\hline First & $48 \pm 5.33$ & $113.4 \pm 12.20$ & $441 \pm 53.2$ & $20 \pm 4.91$ & $0.8 \pm 0.03$ \\
Second & $49 \pm 5.77$ & $159.2 \pm 11.18^{*}$ & $682 \pm 59.8^{*}$ & $19 \pm 3.24$ \\
\hline
\end{tabular}

Mean values (standard deviation) are shown for the six animals in each group.

*Significant difference $(P<0.05)$.

First group: control group. Second group: B. thuringiensis treatment group.

Histopathological Evaluation

Oral administration of $B$. thuringiensis biopesticide induced histopathological complications such as congestion in the liver and heart. It also caused inflammation and congestion in other vital organs of animals including lung and kidney in the experimented group (5000 mg/kg) (Figure 1).

\section{Body Weight}

Single dose administration of $B$. thuringiensis biopesticide induced a significant decline in the animal body weight mean in the experimental group $(5000 \mathrm{mg} / \mathrm{kg})$ in comparison to the control group (Table 3).

\section{Discussion}

The use of biological insecticides as substitutes for chemical pesticides is a safer alternative for insect control in agricultural crops. ${ }^{13}$ Nevertheless, biological pesticides may induce adverse effects in human, animals, and the environment. ${ }^{14}$

This study is the first investigation to evaluate the potential toxic impacts of $B$. thuringiensis microbial pesticide in experimented animals in Iran. Previous studies have demonstrated the toxicity of Cry toxins to mammals would negligible due to absence known specific receptors in mammalian intestinal cells. ${ }^{15,16}$ Nevertheless, the findings of our study demonstrated that these types of toxins could induce hematotoxicity in experimentally rats. In addition to our findings, other studies also demonstrated some hematological disturbances following $B$. thuringiensis biopesticides exposure in laboratory animals. Mezzomo et al reported the hematotoxicity and genotoxicity of four $B$. thuringiensis spore-crystals genetically modified (GM) to express individually Cry1Aa, CrylAb, CrylAc or Cry2A following single dose administration of $27 \mathrm{mg} / \mathrm{kg}, 136 \mathrm{mg} / \mathrm{kg}$ or $270 \mathrm{mg} / \mathrm{kg}$ in mice. A significant decrease in bone marrow cell proliferation presented cytotoxic but not genotoxic impacts. ${ }^{17}$

The findings of this study demonstrated that the Cry toxins of $B$. thuringiensis could induce hematotoxicity in mice. In this study, Cry 10Aa and Cry 1Ba6 significantly decreased the MCV values, while Crylla increased the red cell distribution width above the reference values. Also, Cry1Ba6 and Cry1Ba6 significantly reduced the number of neutrophils and monocytes, and Cry1Ba6, which significantly decreased both. ${ }^{18}$ High white blood cell count or leukocytosis can be resulted from some complications such as infection, inflammation, severe allergic reactions, a disease of bone marrow and immune system disorder. ${ }^{19}$
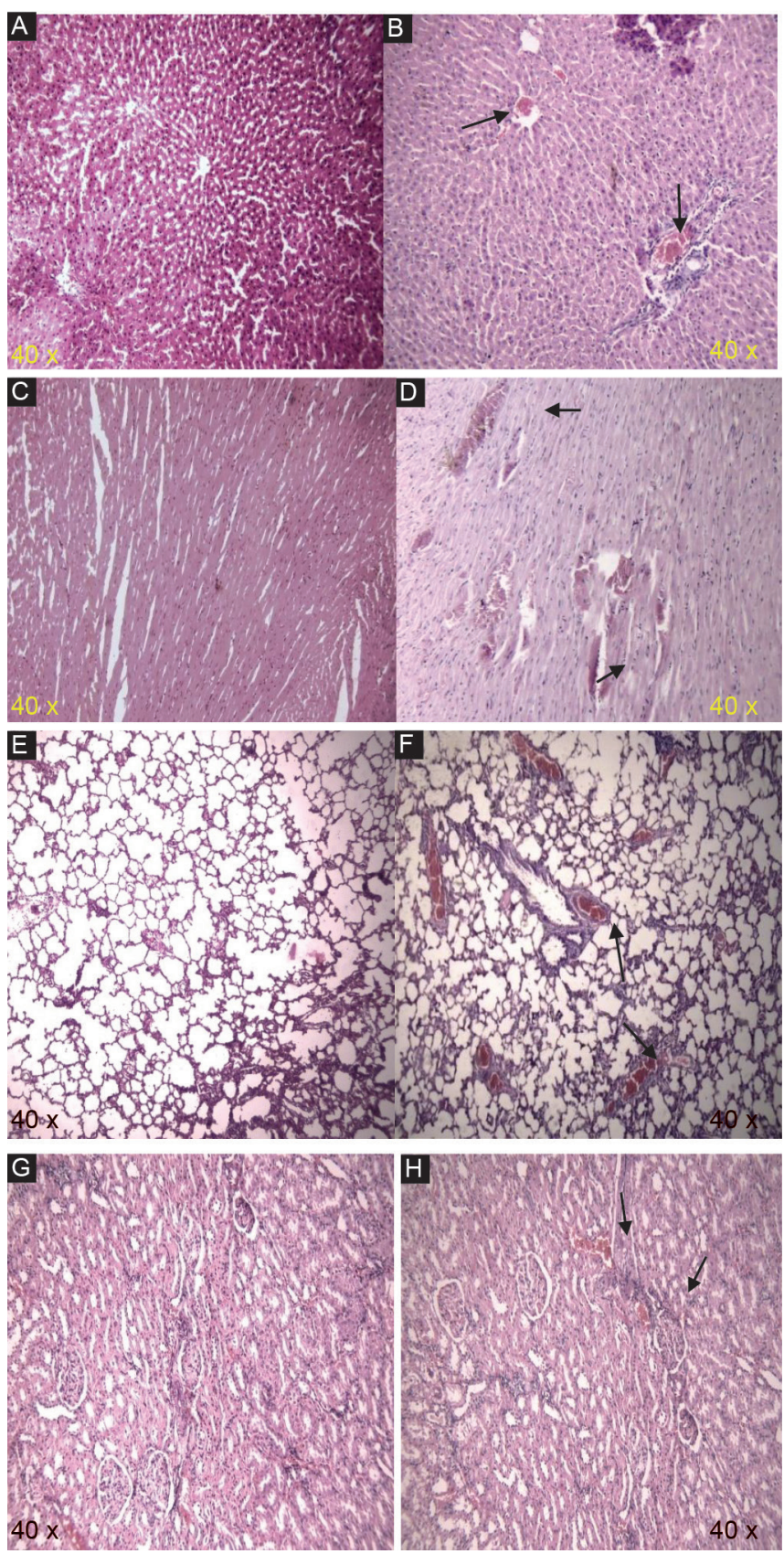

Figure 1. Photomicrographs Of Liver, Heart, Lung And Kidney, sections obtained from rats exposed to Bacillus thuringiensis var kurstaki Biopesticide (5000 mg/kg). (A): normal liver (H\&E), (B): congestion in liver (H\&E), (C): normal heart (H\&E), (D): congestion in heart (H\&E), (E): normal lung $(\mathrm{H} \& \mathrm{E}),(\mathrm{F})$ : congestion and inflammation in lung $(\mathrm{H} \& \mathrm{E}),(\mathrm{G})$ : normal kidney (H\&E), (H): congestion and inflammation in kidney (H\&E). 
Table 3. Body Weight Following Oral Administration of Bacillus thuringiensis var kurstaki Biopesticide $(5000 \mathrm{mg} / \mathrm{kg})$ in Rats

\begin{tabular}{lccc}
\hline Study Groups & $\begin{array}{c}\text { Initial Body } \\
\text { Weight (g) }\end{array}$ & $\begin{array}{c}\text { Final Body } \\
\text { Weight (g) }\end{array}$ & $\begin{array}{c}\text { Body } \\
\text { Weight Difference (g) }\end{array}$ \\
\hline First & $202 \pm 20$ & $253 \pm 23$ & 53 \\
Second & $198 \pm 19$ & $212 \pm 24^{*}$ & 12 \\
\hline
\end{tabular}

Mean values (standard deviation) are shown for the six animals in each group.

* Significant difference $(P<0.05)$.

First group: control group. Second group: B. thuringiensis treatment group.

Platelets are acute-phase reactants, therefore, they are increased in response to various stimuli, including systemic infections, inflammatory conditions, bleeding, and tumors. Actually, this is called reactive or secondary thrombocytosis, which is a benign form of thrombocytosis. Abnormal blood clotting can be dangerous, as blood clots may block the flow of blood to the brain, liver, heart, and other vital organs. ${ }^{20}$ Significant elevation in RBC, WBC and PLT counts was observed in this study. This rise was probably due to the harmful effects of Cry toxins on bone marrow, hematopoietic organs and hematopoietic progenitor cells. On the other hand, the results of this study revealed that oral administration of high dose of this biopesticide induced histopathological alterations such as congestion and inflammation in vital organs of the experimented rats. However, there was no report about pathological changes which can be induced with microbial biopesticides in animals. This is while, a few studies demonstrated inflammation, congestion, edema and hemorrhage in some organs following the administration of some chemical pesticides such as fipronil and chlorpyrifos in animals. $^{21}$

Congestion or hyperemia represents the increase of blood in a territory, due to dilatation of small vessels. According to the mechanism, it may be active or passive. Active congestion is a result of arteriolar distension (e.g., skeletal muscle activity, inflammation, local neuro-vegetative reaction). Passive congestion also termed as stasis, is a consequence of an impaired venous drainage (heart failure, compression or obstruction of veins), that is followed by dilatation of venules and capillaries. ${ }^{22-24}$ However, the mechanism of congestion that was induced by this biopesticide was not clear and needs further investigations. The changing of enzymes in the blood is usually used as a marker for the diagnosis of tissue damages. In the present study, the activity of hepatic biomarkers was significantly raised 2 weeks after oral administration of this biopesticide in animals, while the serum concentration of renal biomarkers did not significantly change. The ALT and AST are mainly found in the liver. ${ }^{25}$ Also, the AST and ALT have been formerly called serum glutamic oxaloacetic transaminase and serum glutamic pyruvic transaminase, respectively. ${ }^{26}$ The AST or ALT serum concentrations are an essential aid in the diagnosis of liver disorders in combination with other markers and histopathology findings. ${ }^{27}$ The AST and ALT levels during liver damages can be elevated 10 to 20 times and up to 50 times compare to normal subjects, respectively. ${ }^{28}$ On the other hand, the ratio of AST to ALT
(AST/ALT) can occasionally help to determine whether the liver has been damaged or not. The ALP is an enzyme for the plasma membrane and endoplasmic reticulum. This biomarker is often used to evaluate the integrity of the plasma membrane. ${ }^{29}$ Significant increment of the serum ALP level is usually noticed in liver damage and heart infections. ${ }^{30,31}$ However, the findings of the present study demonstrated that high doses of this biopesticide might induce hepatoxicity respecting significant increases of ALT, AST and ALP activity in experimental rats. Creatinine and BUN are the final products of protein metabolism and their levels will be increased in renal failure. ${ }^{32}$ Hence, respecting to the results, there is no evidence to nephrotoxicity. Arteaga et al demonstrated that new formulation of $\mathrm{Bti} \mathrm{SH}-14$ is not acutely toxic via dermal route and has no dermal irritation or hypersensitivity but has low eye irritation in the experimented rabbits. ${ }^{33}$ Wilcks et al performed an oral administration of $B$. thuringiensis in rats. ${ }^{34}$ Finally, these bacteria were detected at high density in fecal and intestinal samples even 2 weeks following the last dosage. ${ }^{34}$

Peng et al reported an oral administration of GM $B$. thuringiensis with vegetative insecticidal protein gene in rats. ${ }^{35}$ The results did not show significant differences between control and treated groups following analysis of some factors including body weight gain, food and water consumptions, clinical observations, hematology, serum biochemistry, organ weight ratios and histopathological findings. ${ }^{35,36}$

In the present study, the body weight of the B. thuringiensis treated group of the experimented animals was significantly raised in comparison to the control group. In another study, the acute oral toxicity of GM B. thuringiensis supplemented with an additional $\mathrm{N}$-acyl homoserine lactones gene were studied in Wistar rats. ${ }^{37}$ In contrast with the findings of this study, Meher et al reported that acute oral administration of $B$. thuringiensis in rats has no adverse effects. ${ }^{37}$ Previous studies also demonstrated that different doses of $B$. thuringiensis did not have acute toxicity following the administration in rats. ${ }^{38}$

\section{Conclusions}

In conclusion, findings demonstrated that oral administration of high dose of $B$. thuringiensis var kurstaki biological insecticide can induce some biochemical, hematological and pathological abnormalities in vertebrates such as rats. Incorrect usage of this biological insecticide by farmers can raise the risk of human exposure to significant scale of this biopesticide and can finally cause consequent health problems. The findings propose that further in vivo investigations are needed to characterize the toxicological risks for human beings.

\section{Authors' Contributions}

RAL was involved to carry out experiments and preparation of the manuscript and finalized the manuscript. EZ participated in designing of the study and performed the statistical analysis and data interpretation. $\mathrm{NH}$ and $\mathrm{AA}$ were involved in the manuscript writing and data interpretation.

Conflict of Interest Disclosures

The authors declare that there are no conflicts of interest regarding the 
publication of this manuscript.

\section{Acknowledgments}

This work was financed by Standard Research Institute.

References

1. Sudakin DL. Biopesticides. Toxicol Rev. 2003;22(2):83-90. doi:10.2165/00139709-200322020-00003.

2. Palma L. Bacillus thuringiensis-based biopesticides, are they as effective as they should be? Rev Argent Microbiol. 2017;49(1):119. doi:10.1016/j.ram.2016.10.003.

3. Manns DC, Churey JJ, Worobo RW. Functional assignment of YvgO, a novel set of purified and chemically characterized proteinaceous antifungal variants produced by Bacillus thuringiensis SF361. Appl Environ Microbiol. 2012;78(8):2543-2552. doi:10.1128/ aem.07727-11.

4. Yan M, Roehrl MH, Wang JY. Discovery of crystalline inclusions in Bacillus licheniformis that resemble parasporal crystals of Bacillus thuringiensis. Can J Microbiol. 2007;53(9):1111-1115. doi:10.1139/w07-076.

5. Sauka DH, Benintende GB. [Bacillus thuringiensis: general aspects. An approach to its use in the biological control of lepidopteran insects behaving as agricultural pests]. Rev Argent Microbiol. 2008;40(2):124-140.

6. Burgerjon A. [Physiologic and mutagenic action of the thermostable toxin of Bacillus thuringiensis Berliner on insects]. Ann Parasitol Hum Comp. 1973;48(6):835-844.

7. Ruiz de Escudero I, Estela A, Escriche B, Caballero P. Potential of the Bacillus thuringiensis toxin reservoir for the control of Lobesia botrana (Lepidoptera: Tortricidae), a major pest of grape plants. Appl Environ Microbiol. 2007;73(1):337-340. doi:10.1128/ aem.01511-06.

8. Tanaka S, Endo H, Adegawa S, Kikuta S, Sato R. Functional characterization of Bacillus thuringiensis Cry toxin receptors explains resistance in insects. Febs j. 2016;283(24):4474-4490. doi:10.1111/febs.13952.

9. Rubio-Infante N, Moreno-Fierros L. An overview of the safety and biological effects of Bacillus thuringiensis Cry toxins in mammals. J Appl Toxicol. 2016;36(5):630-648. doi:10.1002/jat.3252.

10. Gomez I, Pardo-Lopez L, Munoz-Garay C, et al. Role of receptor interaction in the mode of action of insecticidal Cry and Cyt toxins produced by Bacillus thuringiensis. Peptides. 2007;28(1):169-173. doi:10.1016/j.peptides.2006.06.013.

11. Shinkawa A, Yaoi K, Kadotani T, et al. Binding of phylogenetically distant Bacillus thuringiensis cry toxins to a Bombyx mori aminopeptidase $\mathrm{N}$ suggests importance of Cry toxin's conserved structure in receptor binding. Curr Microbiol. 1999;39(1):14-20. doi:10.1007/pl00006820.

12. Stalinski R, Laporte F, Despres L, Tetreau G. Alkaline phosphatases are involved in the response of Aedes aegypti larvae to intoxication with Bacillus thuringiensis subsp. israelensis Cry toxins. Environ Microbiol. 2016;18(3):1022-1036. doi:10.1111/14622920.13186.

13. el-Bendary MA. Bacillus thuringiensis and Bacillus sphaericus biopesticides production. J Basic Microbiol. 2006;46(2):158-170. doi:10.1002/jobm.200510585.

14. Prasad SS, Shethna YI. Enhancement of immune response by the proteinaceous crystal of Bacillus thuringiensis var thuringiensis. Biochem Biophys Res Commun. 1975;62(3):517-523. doi:10.1016/0006-291X(75)90429-5.

15. Betz FS, Hammond BG, Fuchs RL. Safety and advantages of Bacillus thuringiensis-protected plants to control insect pests. Regul Toxicol Pharmacol. 2000;32(2):156-173. doi:10.1006/ rtph.2000.1426.

16. Hofte H, Whiteley HR. Insecticidal crystal proteins of Bacillus thuringiensis. Microbiol Rev. 1989;53(2):242-255.
17. Mezzomo BP, Miranda-Vilela AL, Barbosa LC, Albernaz VL, Grisolia CK. Hematotoxicity and genotoxicity evaluations in Swiss mice intraperitoneally exposed to Bacillus thuringiensis (var kurstaki) spore crystals genetically modified to express individually Cry1 Aa, Cry1 Ab, Cry1 Ac, or Cry2Aa. Environ Toxicol. 2016;31(8):970-978. doi:10.1002/tox.22106.

18. de Souza Freire I, Miranda-Vilela AL, Barbosa LC, Martins ES, Monnerat RG, Grisolia CK. Evaluation of cytotoxicity, genotoxicity and hematotoxicity of the recombinant spore-crystal complexes Cry1la, Cry10Aa and Cry1Ba6 from Bacillus thuringiensis in Swiss mice. Toxins (Basel). 2014;6(10):2872-2885. doi:10.3390/ toxins6102872.

19. Abramson N, Melton B. Leukocytosis: basics of clinical assessment. Am Fam Physician. 2000;62(9):2053-2060.

20. Tefferi A, Barbui T. Polycythemia vera and essential thrombocythemia: 2017 update on diagnosis, risk-stratification, and management. Am J Hematol. 2017;92(1):94-108. doi:10.1002/ ajh.24607.

21. Barfod KK, Poulsen SS, Hammer M, Larsen ST. Sub-chronic lung inflammation after airway exposures to Bacillus thuringiensis biopesticides in mice. BMC Microbiol. 2010;10:233. doi:10.1186/1471-2180-10-233.

22. Goyal P, Sehgal S, Ghosh S, et al. Histopathological correlation of atypical (c3) and suspicious (c4) categories in fine needle aspiration cytology of the breast. Int J Breast Cancer. 2013;2013:965498. doi:10.1155/2013/965498.

23. Gambhir MS, Goyal SK, Rawat ML. Hepatic involvement in abdominal tuberculosis (a clinical, biochemical and histopathological study). J Assoc Physicians India. 1972;20(11):843847.

24. Acharya S, Goyal A, Rattan V, Vaiphei K, Kaur Bhatia S. Dentigerous cyst or adenomatoid odontogenic tumor: clinical radiological and histopathological dilemma. Case Rep Med. 2014;2014:514720. doi:10.1155/2014/514720.

25. Asgharnia M, Mirblouk F, Salamat F, Ashrafkhani B, Dirbaz Z. Predictive value of aspartate aminotransferase and alanine aminotransferase levels in vaginal fluid for the diagnosis of premature rupture of membranes. Iran J Reprod Med. 2014;12(4):269-274.

26. Gurung RB, Purbe B, Gyawali P, Risal P. The ratio of aspartate aminotransferase to alanine aminotransferase (AST/ALT): the correlation of value with underlying severity of alcoholic liver disease. Kathmandu Univ Med J (KUMJ). 2013;11(43):233-236. doi:10.3126/kumj.v11i3.12511.

27. Nanji AA, French SW, Freeman JB. Serum alanine aminotransferase to aspartate aminotransferase ratio and degree of fatty liver in morbidly obese patients. Enzyme. 1986;36(4):266-269. doi:10.1159/000469304.

28. Magarlamov AG. [Aspartate and alanine aminotransferase activity in the liver and blood serum of protein-deficient rats receiving parenteral nitrogen nutrition]. Ukr Biokhim Zh (1978). 1980;52(6):720-726.

29. Zhou YJ, Zou H, Zheng JN, et al. Serum alkaline phosphatase, a risk factor for non-alcoholic fatty liver, but only for women in their 30s and 40s: evidence from a large cohort study. Expert Rev Gastroenterol Hepatol. 2017;11(3):269-276. doi:10.1080/174741 24.2017.1283984.

30. Wannhoff A, Rauber C, Friedrich K, et al. Von Willebrand factor and alkaline phosphatase predict re-transplantation-free survival after the first liver transplantation. United European Gastroenterol J. 2017;5(1):86-93. doi:10.1177/2050640616650060.

31. Gevers TJ, Nevens F, Torres VE, Hogan MC, Drenth JP. Alkaline phosphatase predicts response in polycystic liver disease during somatostatin analogue therapy: a pooled analysis. Liver Int. 2016;36(4):595-602. doi:10.1111/liv.12986. 
32. Stark JL. BUN/creatinine: your keys to kidney function. Nursing. 1980;10(5):33-38.

33. Arteaga ME, Mancebo A, Molier T, et al. Dermal toxicity, eye and dermal irritation and skin sensitization evaluation of a new formulation of Bacillus thuringiensis var israelensis $\mathrm{SH}-14$. Regul Toxicol Pharmacol. 2014;68(1):147-151. doi:10.1016/j. yrtph.2013.12.003.

34. Wilcks A, Hansen BM, Hendriksen NB, Licht TR. Persistence of Bacillus thuringiensis bioinsecticides in the gut of human-floraassociated rats. FEMS Immunol Med Microbiol. 2006;48(3):410418. doi:10.1111/j.1574-695X.2006.00169.x.

35. Peng D, Zhou C, Chen S, Ruan L, Yu Z, Sun M. Toxicological safety assessment of genetically modified Bacillus thuringiensis with additional $\mathrm{N}$-acyl homoserine lactonase gene. Environ Toxicol
Chem. 2008;27(1):188-195. doi:10.1897/07-059.1.

36. Peng D, Chen S, Ruan L, Li L, Yu Z, Sun M. Safety assessment of transgenic Bacillus thuringiensis with VIP insecticidal protein gene by feeding studies. Food Chem Toxicol. 2007;45(7):1179-1185. doi:10.1016/j.fct.2006.12.026.

37. Meher SM, Bodhankar SL, Arunkumar, Dhuley JN, Khodape DJ, Naik SR. Toxicity studies of microbial insecticide Bacillus thuringiensis var. kenyae in rats, rabbits, and fish. Int J Toxicol. 2002;21(2):99-105. doi:10.1080/10915810252866079.

38. Carter JN, Liggett MP. Acute oral toxicity and infectivity/ pathogenicity to rats of EG 7841. England: Huntingdon Research Centre Ltd, Huntingdon, Cambridgeshire; 1994. HRC Study Report No ECO 6/942538. 